Joachim Perels

\title{
Diether Posser - Anwalt des Rechtsstaats in restaurativen Zeiten*
}

"Viele, die ihrer Zeit vorausgeeilt waren, mußten auf sie in sehr unbequemen Unterkünften warten. «Dieser Aphorismus Stanislaws Jerzy Lec' hat Gültigkeit für die Rolle Diether Possers in der Ära Adenauer. Geistiges Vorauseilen bedeutete, unter den Bedingungen der zugespitzten Ost-West-Konfrontation unbeirrt rechtsstaatlich zu handeln. Arnold Freymuth war in seiner Auseinandersetzung mit der Landesverratsjustiz der Weimarer Republik damit konfrontiert, daß - in seinen Worten - „die Richter von heute noch die des alten Systems sind «'. Ein vergleichbares Problem belastete die frühe Bundesrepublik: die weitgehende - nur durch das Bundesverfassungsgericht unterbrochene - Kontinuität des Justizapparates der NS-Diktatur. "Der große Defekt des deutschen Beamtensystems " (und, wie sich hinzufügen läßt, der Justiz) »liegt«, wie der deutsche Emigrant Arnold Brecht zu Beginn der soer Jahre formulierte, »im Konflikt zwischen der autoritären Tradition und der bürokratischen Struktur auf der einen Seite und den wesentlichen Prinzipien einer demokratischen Gesellschaft auf der anderen. «²

Diese Konstellation wurde durch den Kalten Krieg, die Logik der Blockkonfrontation, verschärft. Sie setzte sich bis in den Gerichtssaal fort - entsprechend dem Wort des Abgeordneten Haasler (CDU/CSU) von 1957, daß das Politische Strafrecht eine "Waffe« sei, "die geschmiedet wurde, um im Kalten Krieg zu bestehen «3

Diether Posser entwickelte zu Beginn der soer Jahre eine grundlegende, zunächst politische Kritik am Schematismus des Kalten Krieges. Eine gewisse Rolle mag dabei gespielt haben, daß ein Major der Roten Armee ihn, den Offizier der Deutschen Wehrmacht, bei Kriegsende vor der Erschießung bewahrte. Posser betrachtete die Sowjetunion und die von ihr abhängigen Staaten nicht als »Todfeinde« (Adenauer), sondern lediglich als Gegner. Kommunistische Regierungspolitik wurde nach dem Zweiten Weltkrieg in ein Freund-Feind-Schema eingeordnet, die Erledigung des Feindes zum strategischen Ziel erklärt. In dieser Situation entwickelte Posser Anfang der soer Jahre, als Geschäftsführer der "Notgemeinschaft zur Rettung des Friedens in Europa « und publizistischer Kopf der wenig später gegründeten "Gesamtdeutschen Volkspartei« Gustav Heinemanns und Helene Wessels, eine Position, die, lange vor der neuen Ostpolitik, die Möglichkeiten auch der Kooperation mit der sowjetischen Großmacht auszuloten suchte, ohne die - das wurde seinerzeit fast verdrängt das NS-Regime nicht hätte zerschlagen werden können.

Die auf die Herstellung geregelter Konfliktbeziehungen zwischen Ost und West gerichtete Perspektive Possers entsprang nicht allein einem moralischen Postulat. Sie

* Vorrrag aus Anlaß der Verleıhung des Arnold-Freymuth-Preıses 1994 an Dr. Diether Posser am 27. 11. 1994 in Hamm. Die Arnold-Freymuth-Gesellschaft e. V. (Marker Allee 48, 59063 Hamm) sucht demokratisches Engagement in der Justız zu starken. Die Gesellschaft erinnert an einen der wenigen republikanischen Vertreter der Justız in der ersten deutschen Demokratie. Arnold Freymuth war lange Jahre Senatsprasident am Kammergerıcht. Kurz nach der Errichtung der NS-Diktatur nahm er sich im Pariser Exil das Leben. Der Vortrag wurde fur die Druckfassung uberarbettet.

1 Vorwarts 28.4.1922 zit. nach J.H.Luth/U. Wesel, Arnold Freymuth, Hermann Großmann, Alfred Orgler. Drei Richter fur die Republik, in: Kritısche Justız (Hg.), Streitbare Jurısten, Baden-Baden 1988, S. 2 II, s. auch O. Jung, Senatsprasident Freymuth, Frankfurt/M. 1989.

2 A. Brecht, Personnel Management, in: E. H. Litchfield (Hg.), Governing Postwar Germany, Ithaca I953, S. 263 ff. (292).

3 Bundestagsprotokoll vom 8. Februar 1957, S. 10931. 
gründete sich auf eingehende völkerrechtliche Studien zur Fortgeltung der Kontrollratskompetenz, die einen separaten westdeutschen Wehrbeitrag ausschloß. Sie beruhte zudem auf einer Aufarbeitung der Geschichte der Sowjetunion, die die Politik der UdSSR entdämonisierte und ihre Interessenlagen als Kalkül für eine mögliche Kooperationspolitik vor allem in der Frage der Wiedervereinigung analysierte. ${ }^{4}$ Wer sich das Gewicht der überwiegend restaurativen Justiz - fast ausnahmslos legitimierte sie die Rechtsordnung des Nationalsozialismus - und die polarisierten Strukturen des Kalten Krieges vor Augen führt, kann ermessen, welchen geistigen Barrieren sich Diether Posser als Anwalt in politischen Prozessen gegen Kommunisten gegenübersah.

Er konnte seine juristisch-praktische Tätigkeit wohl nur durchhalten, weil er sie immer auch im politischen Kontext der Überwindung des Kalten Krieges im gespaltenen Deutschland begriff. Deutlich wird dies in einem Beweisantrag, der ihm - so waren damals die Zeiten - ein erst nach langem Hin und Her eingestelltes Ehrengerichtsverfahren eintrug. In dem Antrag hatte Posser nachgewiesen, daß Bundeskanzler Adenauer in einer Stellungnahme vor dem Bundestag die Noten der Sowjetunion von I952, die für den Fall eines militärisch neutralen Status von Gesamtdeutschland eine Wiedervereinigung auf demokratischer Grundlage anboten, objektiv wahrheitswidrig dargestellt hatte.

Neben dem politischen Motiv spielte für Posser ein christlicher Beweggrund eine Rolle. Gustav Heinemann, in dessen Anwaltskanzlei er Anfang der soer Jahre als Sozius eintrat, wurde sein Mentor. Heinemann hatte die vom Rat der Evangelischen Kirche abgegebene Stuttgarter Schulderklärung von 1945 mitunterzeichnet, in der der für die Aussöhnung mit dem Osten wichtige Satz stand: »Durch uns ist unendliches Leid über viele Völker und Länder gebracht worden «. In Heinemanns Person waren die Lehren der Bekennenden Kirche, ihr Versuch, für das Recht der Verfolgten einzutreten, auch für Posser präsent. Posser war von jenem Pfarrer Graeber konfirmiert worden, der seinerzeit auch Heinemanns Hinwendung zu einem aktivpraktischen Christentum ausgelöst hatte. Possers innere Unabhängigkeit hatte wohl mit einem Wort Gustav Heinemanns zu tun, dem er seine Erinnerungen »in dankbarer Verehrung" gewidmet hatte. Das Wort lautet: „Die Herren dieser Welt gehen, unser Herr kommt."

Als Anwalt entwickelte Posser ein strategisch durchdachtes Arsenal von rechtsstaatlichen Argumentationen, um die Praxis politischer Justiz von der Ausgrenzungslogik des Kalten Krieges zu trennen. Dies geschah auf drei miteinander verknüpften Ebenen.

Zum einen kritisierte Posser das rechtsstaatliche Defizit des I. Strafrechtsänderungsgesetzes von 1951, das die Verfolgung von Kommunisten im Kalten Krieg regelte. Zum anderen versuchte er in einer gemeinsam mit Rechtsanwalt Ammann verfaßten Denkschrift, die Wirkung des "politischen Strafrechts neuer Art " (Copic) durch die auch von der FDP, der SPD und der Evangelischen Kirche vertretene Forderung nach einer Amnestie mehrheitsfähig zu machen. Sein wichtigstes Tätigkeitsfeld aber bestand in einer Fülle minuziös vorbereiteter Prozesse (gegen Mitglieder der Gesellschaft für Deutsch-Sowjetische Freundschaft, der Organisation Frohe Ferien für alle Kinder, von Mitgliedern des westdeutschen Friedenskomitees, kommunistischer

4 Vgl. D. Posser, Deutschlands Rechtslage und die Verteidigung der Bundesrepublik, Stimme der Gemeinde H. ${ }_{2} / 1951$, S. 4 f., ders., Recht ist, was uns nützt? Stimme der Gemeinde H. $3 / 1952$, Sp. 89 ff., ders., Weiß die Mehrheit im Parlament, was sie tut? Stimme der Gemeinde H. 5/1952, Sp. 137 f., ders., Deutschsowjetische Beziehungen (1955), 2. Aufl. Darmstadt 1963.

5 D. Posser, Anwalt im Kalten Krieg. Ein Stuck deutscher Geschichte in Politischen Prozessen, München 1991, S. 129 ff. 
Einzelkandidaten und vieler anderer mehr). Sein Ziel war, der Gefahr entgegenzutreten, "daß die Handlungen der Angeklagten (nicht) Gegenstand eines juristischen Urteils, sondern die Angeklagten Opfer eines politisch-weltanschaulichen Vorurteils wurden ${ }^{6}{ }^{6}$

Der Konstruktionsfehler des 1. Strafrechtsänderungsgesetzes lag für Posser darin, $\mathrm{daß}$ es in zentralen Bestimmungen nicht konkrete Handlungen und Rechtsgutverletzungen, sondern Gesinnungen zum Anknüpfungspunkt von Strafsanktionen machte. Dieser bereits für die antiliberale Strafrechtsdoktrin des NS-Systems konstitutive Bezugspunkt erweiterte - über ein rechtsstaatliches Maß hinaus - die Zugriffskapazität des Staates auf die Individuen. An zwei Normen führte Posser dies in einer Broschüre von I96I näher aus:

»... Bedenklich sind die Staatsschutznormen, deren objektiver Tatbestand keinerlei Unrechtsgehalt besitzt, sondern ihre strafrechtliche Kennzeichnung erst durch eine bestimmte Absicht des Täters erhalten, nämlich die Absicht, >den Bestand oder die Sicherheit der Bundesrepublik Deutschland zu beeinträchtigen, einen der in $\$ 88$ bezeichneten Verfassungsgrundsätze zu beseitigen, außer Geltung zu setzen oder zu untergraben $(\mathbb{S} 92$, rood Abs. 2). Das Unrechtmäßige der strafrechtlich wertneutralen Handlung wird allein durch die an das Handeln geknüpfte Absicht begründet, also durch ein rein inneres, mit Beweismitteln höchst unzulänglich feststellbares Element. «7

Possers Auseinandersetzung mit den neuen Staatsschutznormen blieb bis auf eine Ausnahme erfolglos. Eine Amnestie für politische Straftäter war 1957 am Widerstand der Regierung Adenauer gescheitert, obgleich dank ihrer Mitwirkung ein Klima möglicher Amnestie für NS-Gewalttäter geschaffen wurde. Posser gelang es, wiederum gemeinsam mit Rechtsanwalt Ammann, eine Schlüsselnorm des Politischen Strafrechts, $\$ 90$ a Abs. 3 StGB, den insbesondere der Bundesgerichtshof ständig anwandte, vom Bundesverfassungsgericht für verfassungswidrig erklären zu lassen. ${ }^{8}$ Nach dieser Norm konnten, unter Verletzung des Rückwirkungsverbots, Mitglieder der KPD für Handlungen bestraft werden, die das Verbot der KPD voraussetzten. In der Verfassungsgeschichte der Bundesrepublik ist kaum mehr im Bewußtsein, wer diese für die Grenzziehung der Strafgewalt des Staates wegweisende Entscheidung erstritten hat.

Insgesamt erfolgten nach den Berechnungen von v. Brünneck von I95 I bis 19666758 Verurteilungen, zugleich wurden von I95 I bis I968 I 38000 Ermittlungsverfahren auf der Grundlage des neuen Politischen Strafrechts durchgeführt. ${ }^{9}$ In den vielen Verfahren, an denen Posser als Anwalt beteiligt war, durchbrach er das FreundFeind-Schema aus rechtsstaatlichen Gründen: Handlungen von Kommunisten dürfen nur nach dem objektiven Unrechtsgehalt beurteilt werden, nicht danach, ob die Angeklagten subjektiv Kommunisten seien. Das Landgericht Düsseldorf hingegen, vor dem Posser 1960 Vertreter des westdeutschen Friedenskomitees verteidigte, setzte dem anwaltlichen Argument, Vertreter des Komitees hätten lediglich dieselben oder vergleichbare Ansichten geäußert wie andere kritische Vertreter der Öffentlichkeit, die These entgegen: „In verfassungstreuer Absicht geäußerte politische Werturteile sind strafrechtlich wertneutral ${ }^{10}{ }^{10}$ Das bedeutete: Bei einem unterstellten

\footnotetext{
6 Ebd., S. 299.

7 D. Posser, Politısche Strafjustiz aus der Sicht des Vertesdigers, Karlsruhe 196r, S. 8.

8 BVerfGE 12, S. $296 \mathrm{ff}$. s. dazu D. Posser, Anwalt im Kalten Krieg (Anm. 5), S.219ff.

9 A.v. Brunneck, Politische Justiz gegen Kommunisten in der Bundesrepublik Deutschland 1949-1968, Frankfurt/M. 1978, S. 278 .

10 D. Posser, Politische Strafjustiz (Anm. 7), S. 4 I s. auch R. Lang (= D. Posser), Der Dusseldorfer Prozeß, Sonderdruck der Stimme der Gemeinde H. 10-13/1960.
} 
Fehlen einer verfassungstreuen Absicht können die inhaltlich gleichen politischen Werturteile strafrechtlich relevant werden - ein rechtsstaatlich inakzeptables Ergebnis, weil damit gleiche Handlungen strafrechtlich ungleich qualifiziert werden.

Auch in aussichtsloser Lage gab Posser seinen unterkühlten, gelegentlich mit einem Schuß Humor versehenen A rgumentationsduktus nicht auf. Darin schwang offenbar die Hoffnung mit, dem gerichtlichen Gegenüber - und im Falle des Senatspräsidenten am BGH, Heinrich Jagusch, scheint ihm dies in langen Gesprächen auch gelungen zu sein - die Augen für die Humanität des Rechtsstaates gegenüber politischen Gegnern zu öffnen. Wie ein Plädoyer, in dem die Achtung für das politische Schicksal von Kommunisten mitschwingt, die damals bis in die Schulbücher hinein kaum zu finden war, liest sich ein Abschnitt aus Possers schon erwähnter Broschüre zur Politischen Strafjustiz von I96r:

»Als Rädelsführer der KPD nach $\$ 90$ a und $\$ I 29$ wurde ein Redakteur an einer kommunistischen Tageszeitung verurteilt, der nicht einen einzigen Artikel mit beleidigendem Inhalt geschrieben hatte, nicht vorbestraft war und einen vorzüglichen Leumund besitzt. In dem Urteil wird über seinen Werdegang folgendes festgestellt: >1933 studierte er an der Universität Köln. Dort wurde er wegen seiner jüdischen Abstammung exmatrikuliert. Im selben Jahr noch emigrierte er nach Frankreich, von wo er sich 1934 nach Holland begab... 1935 machte er sich in Amsterdam selbständig... Dieses Geschäft wurde 1942 liquidiert. Im darauffolgenden Jahr tauchte er sodann mit einem Teil seiner Familie bis Kriegsschluß unter ... Das älteste Kind, eine Tochter, ist jetzt I 8 Jahre alt. Diese mußte der Angeklagte drei Monate nach ihrer Geburt aus Sicherheitsgründen unter falschem Namen in ein Waisenhaus geben. Seine Eltern sind im KZ Bergen-Belsen verhungert. 29 andere Verwandte wurden in Auschwitz und anderen Vernichtungslagern vergast... Nach dem Zusammenbruch kehrte er 1945 nach Deutschland zurück und fand Beschäftigung bei der Bezirksleitung der KPD in Köln. 1946 kam er als Redakteur zur >Neuen Volkszeitungく. Dort war er bis zum Verbot der KPD am 17.8. 1956 tätig<.

Ist wirklich anzunehmen, daß ein Mann, der mit seiner Familie so schreckliche Erfahrungen mit einem Gewaltregime gemacht hat, ’den Boden für die Errichtung einer Gewalt- und Willkürherrschaft vorbereiten len Untergrundorganisation sein wollte? Wie sollte er erkennen, daß bei seiner I0 Jahre (1946-1956) ausgeübten Tätigkeit plötzlich ab 1.9. 195 I - Inkrafttreten des Strafrechtsänderungsgesetzes - straffreies Tun strafbar geworden sein sollte?

Es steht freilich außer Zweifel, daß die KPD in den Jahren I95 I/I955 die Bundesregierung mit größter Schärfe und in beleidigender Weise angegriffen hat. Die dafür Verantwortlichen konnten belangt werden und sind auch wegen Beleidigung, übler Nachrede, Verunglimpfung von Staatsorganen verurteilt worden. Aber kann deswegen eine ganze Partei mit zehntausenden von Mitgliedern zur kriminellen Organisation erklärt werden? स $^{1 \top}$

Der wichtigste Prozeß, in dem es Posser und Heinemann gelang, rechtsstaatliche Grundsätze in vollem Maße durchzusetzen, war das 1957 durchgeführte Verfahren gegen Viktor Agartz, den früheren Leiter des Wirtschaftswissenschaftlichen Instituts des DGB. Die Ausgangslage war eine andere. Agartz war kein Kommunist, sondern ein Sozialist. Ihm wurde angelastet, daß er sich seine Zeitschrift WISO z. T. aus der DDR finanzieren ließ. Als wichtig erwies sich die - auch in der Frankfurter Allgemeinen Zeitung positiv herausgestellte - Zeugenaussage von Professor Wolfgang Abendroth, der, aus der SBZ geflohen, die von Agartz vertretene Position eines demokratischen Marxismus von der stalinistischen Herrschaftsideologie systema-

I I D. Posser, Politische Strafjustız (Anm. 7), S. ${ }_{3}$ f. 
tisch unterschied. Vor allem aber die brillant rechtsstaatlichen, noch heute lesenswerten Plädoyers von Heinemann und Posser ${ }^{12}$ bewirkten, daß der Bundesgerichtshof Agartz vom Vorwurf landesverräterischer Beziehungen zum FDGB der DDR freisprach.

Die Entscheidung blieb eine Ausnahme. Possers Resümee war, daß »die Straftatbestände der Staatsgefährdung Elemente des Gesinnungsstrafrechts enthielten, weil für ein und dasselbe Geschehen Kommunisten bestraft, aber Nichtkommunisten freigesprochen wurden $\ll{ }^{13}$ Der kleine Kreis der Verteidiger in politischen Strafsachen führte lange Jahre fast ein Katakombendasein ohne öffentliche Resonanz, ohne die Möglichkeiten der Publikation in juristischen Fachzeitschriften. All das hatte nicht zur Folge, daß Posser sich in die Resignation treiben ließ.

Der Ton seiner Texte blieb unverändert. 1959 endlich, sieben Jahre nach der Schaffung des I.Strafrechtsänderungsgesetzes, konnte in der NJW ein grundlegender Aufsatz zur Fragwürdigkeit der Politischen Justiz erscheinen. ${ }^{54}$ Der Grund war, daß Heinemann, seit 1957 Bundestagsabgeordneter der SPD, die Untersuchung mitzeichnete. Da die Rechtsprechung gegenüber Kommunisten, trotz der größeren Möglichkeit juristischer Fachkritik, sich kaum veränderte, suchte Posser die politische Öffentlichkeit zum Resonanzboden für die Problematisierung der Politischen Justiz zu machen. Der Durchbruch gelang ihm mit einem Aufsatz in der »ZEIT « von I 963 zur Verhaftung des Chefredakteurs des Deutschlandsenders, Grasnick's, der zum - Aufsehen erregenden - Opfer der systematisch sanktionierten politischen Kontakte zwischen Funktionären der DDR und Bürgern der Bundesrepublik wurde. Posser vertrat Grasnick und erreichte, auch auf Grund der breiten Kritik der Öffentlichkeit, daß der Chefredakteur des Deutschlandsenders aus der Untersuchungshaft entlassen und so ein Zeichen für die allmähliche Veränderung der Politischen Justiz gesetzt wurde.

Da Posser in der Bundesrepublik Kommunisten verteidigte, besaß er besonderes Gewicht, wenn es darum ging, gegen Terrorpraktiken der DDR vorzugehen. Auch gegenüber der DDR wollte Posser das Freund-Feind-Schema durchbrechen, das die Auslieferung der Menschen an eine unbegrenzte Staatsgewalt ermöglicht. Über viele Jahre kämpfte er für die Freilassung des in die DDR verschleppten, nahezu 6 Monate ohne Kontakt zur Außenwelt inhaftierten Gewerkschaftsfunktionärs Heinz Brandt, eines profilierten Anti-Stalinisten. Wenig Erfolg versprachen dabei juristische Instrumentarien. Ein Gespräch Possers mit der Justizministerin der DDR, Hilde Benjamin, blieb erfolglos, worüber er sich - was sonst selten vorkam - in der Unterredung ereiferte. ${ }^{16}$ Angesichts des durch Rechtsgarantien prinzipiell unbegrenzten Machtsystems der DDR setzte Posser Anfang der 6oer Jahre eine andere Strategie ein und übte mit - von ihm angeregten - Interventionen von Herbert Mochalski, Erich Fromm und Bertrand Russell Druck auf die DDR-Spitzen einschließlich Walter $U l$ brichts aus. Drei Jahre nach seiner Verhaftung kam Heinz Brandt, Opfer des von der DDR betriebenen Kalten Krieges, endlich frei.

1968 schloß sich der Kreis. Als die Umorientierung der Außenpolitik gegenüber der Sowjetunion und der DDR unter dem neuen Außenminister Brandt in der Großen Koalition erste Formen annahm, konnte die justizielle Verfolgung von Kommuni-

12 G. Heinemann, Pladoyer, D. Posser, Pladoyer, 1n: H.-G. Hermann, Verraten und Verkauft, Fulda 1959, S. 229 ff., S. $251 \mathrm{ff}$.

13 D. Posser, Anwalt im Kalten Krieg (Anm. 5), S. 297.

14 G. Heinemann/D. Posser, Kritısche Bemerkungen zum politischen Strafrecht in der Bundesrepublik, NJW 1959, S. 121 ff.

is D. Posser, Das Fragwurdige am Fall des Dr. Grasnick, Die Zett v. 21.6.1963, S. 2.

16 D. Posser, Anwalt im Kalten Krieg (Anm. 5), S. 327. 
sten durch die Aufhebung der wichtigsten Bestimmungen des I. Strafrechtsänderungsgesetzes eingestellt werden. Possers Kampf gegen das politische Gesinnungsstrafrecht gelangte spät, für die meisten Betroffenen zu spät, ans Ziel.

Heute steht zu Recht die juristische Aufarbeitung der Regierungskriminalität der DDR auf der Tagesordnung. In dieser Situation besteht die Gefahr eines selbstgerechten Blicks auf die Justizgeschichte der Bundesrepublik, die keine staatliche Kriminalität erzeugt hat. Dennoch muß daran erinnert werden, daß nicht nur Kommunisten der DDR Unrecht begingen, sondern daß auch Kommunisten - freilich in qualitativ unvergleichbarer und im Strafmaß begrenzter Form - durch die Justiz der frühen Bundesrepublik Unrecht erlitten.

Zum Schluß äußere ich eine Bitte. Es wäre gut, wenn nach den Erinnerungen von Posser seine oftmals an abgelegener Stelle veröffentlichten und z.T. auch nicht veröffentlichten juristischen Arbeiten - die Analyse des KPD-Verbotsurteils, das Plädoyer im Agartz-Prozeß, der Bericht über den Düsseldorfer Prozeß etc. ${ }^{17}$ - erneut publiziert würden. Von Possers auf keinem Auge blinden, rechtsstaatlichen Paradigma können die Justiz, die Anwaltschaft und die Rechtslehre bis heute lernen. Das hat auch damit zu tun, daß Possers juristisches Eintreten für politische Außenseiter der Gesellschaft sich nicht in der Analyse erschöpfte, sondern sich im praktischen Handeln - das schon in der Bibel ein größeres Gewicht hat als die selige Kontemplation - bewährte.

\section{Stephan Seltenreich Zur Volkswahl des Bundespräsidenten}

\section{A. Einleitung}

Am 23. Mai 1994 hat die zehnte Bundesversammlung Prof. Dr. Roman Herzog im dritten Wahlgang mit 696 Stimmen zum neuen Bundespräsidenten gewählt. Dabei geben sowohl die Vorgänge im Vorfeld der Wahl als auch zwischen den einzelnen Wahlgängen zu denken. Was ist geschehen? Zunächst schlug der Bundeskanzler den sächsischen Justizminister Steffen Heitmann zum Bundespräsidentschaftskandidaten der Union vor. Doch der war schon innerparteilich nicht zu halten und wurde nachdem die SPD den nordrhein-westfälischen Ministerpräsidenten Johannes Rau als Gegenkandidaten nominiert hatte - gegen den Präsidenten des Bundesverfassungsgerichts Roman Herzog ausgetauscht, der sich einer höheren inner- und teilweise auch überparteilichen Akzeptanz erfreute. Während Bündnis 9o/Die Grünen den parteilosen Bürgerrechtler Jens Reich zu ihrem Kandidaten kürten, trat für die FDP deren langjährige Bundestagsabgeordnete Hildegard Hamm-Brücher an, wobei die FDP-Fraktion sogleich ankündigte, daß Frau Hamm-Brücher bei der Notwendigkeit eines dritten Wahlgangs zurücktreten werde, um eine eindeutige Entscheidung zwischen den beiden "Spitzenkandidaten « Herzog und Rau zu ermöglichen.

Nachdem in den ersten beiden Wahlgängen erwartungsgemäß kein Kandidat die notwendige absolute Mehrheit von $66_{3}$ Stimmen auf sich vereinigen konnte, zogen

17 D. Posser, Polttik und Justız. Ein Wort zum Verbot der KPD und zur politischen Justiz, Stimme der Gemeinde H. 17/1956, Sp. 25 ff., ders., Pladoyer (Anm. 12), ders., Der Dusseldorfer Prozeß (Anm. Io). 